\title{
Time to establish pillars in point-of-care ultrasound
}

\author{
Carolynne J. Cormack ${ }^{1}$ (D) Anthony M. Wald ${ }^{2}$, Peter R. Coombs ${ }^{1,3}$, Leah Kallos ${ }^{1}$ and Gabriel E. Blecher ${ }^{4,5}$ iD \\ ${ }^{1}$ Monash Imaging, Monash Medical Centre, Monash Health, Melbourne, Victoria, Australia \\ ${ }^{2}$ Monash Cardiovascular Research Centre, MonashHeart, Monash Medical Centre, Melbourne, Victoria, Australia \\ ${ }^{3}$ Department of Medical Imaging and Radiation Sciences, Monash University, Melbourne, Victoria, Australia \\ ${ }^{4}$ Monash Emergency Research Collaborative, School of Clinical Sciences at Monash Health, Monash University, Clayton, Victoria, \\ Australia \\ ${ }^{5}$ Monash Medical Centre, Emergency Program, Monash Health, Melbourne, Victoria, Australia
}

\begin{abstract}
Point of care ultrasound (PoCUS) has evolved rapidly and is used by many medical specialties. We propose five essential pillars of PoCUS that are necessary framework for hospital-based PoCUS training and credentialing programs. The pillars are: governance, infrastructure, administration, education and quality. It is time to establish these pillars to ensure the best practice in PoCUS use.
\end{abstract}

Keywords: education, governance, PoCUS, point-of-care ultrasound, quality.

\section{Introduction}

Point-of-care ultrasound (PoCUS) has evolved rapidly and been adopted by many medical specialties. ${ }^{1-4}$ There are significant benefits in both rapid bedside ultrasound diagnosis and safer procedural guidance. ${ }^{1-5}$ However comprehensive PoCUS frameworks are essential to ensure the highest standards of clinical quality and safety. ${ }^{5-7}$ Emphasis is frequently placed upon PoCUS education, without adequate governance, infrastructure, administration and quality assurance processes put in place to support the education. We propose five essential pillars of PoCUS, based upon experience establishing a collaborative PoCUS program for physicians in a large tertiary teaching hospital network. ${ }^{8}$ The five pillars defined are governance, infrastructure, administration, education and quality (Figure 1).

\section{Discussion}

\section{Pillar 1: Governance}

Governance groups are responsible for overseeing the remaining pillars of infrastructure, administration, education and quality. Adequate governance and oversight are essential to ensure clinical excellence and patient safety. ${ }^{5-7}$ Hospital executive endorsement ensures programs are supported operationally and medicolegally. Executive support is also necessary for budget provision for costs associated with a PoCUS program.

Correspondence to email Carolynne.Cormack@monashhealth.org doi: 10.1002/ajum.12126
Governance should be established and managed by key hospital personnel. A PoCUS governance or steering group need not necessarily comprise those most highly skilled in ultrasound but may include program leaders, departmental directors, clinical ultrasound leads, senior clinicians, sonographers, radiologists, cardiologists and medical educators. This coalescence of expertise ensures appropriate scope of practice, educational delivery, necessary infrastructure and clinical quality. ${ }^{8}$ Consideration of scopes of practice, integration of PoCUS findings into clinical management, review of relevant college guidelines, quality audit review, policy development and documentation are among the many essential functions of governance or steering groups. ${ }^{5,6}$

\section{Pillar 2: Infrastructure}

PoCUS requires a planned infrastructure at the point of imaging, and for scan documentation and archive. Moore's law of technological progression is true of the recent evolution of ultrasound machines. ${ }^{9}$ Rapid advancements in ultrasound have facilitated units becoming smaller, lighter, cheaper and more intuitively designed. PoCUS equipment should be appropriate for the clinical context and examination types being performed. A range of equipment is available, from handheld devices with a single transducer through to larger systems capable of comprehensive examinations with full functionality. Equipment purchasing should include consideration of diagnostic capability, image quality, transducers, equipment 


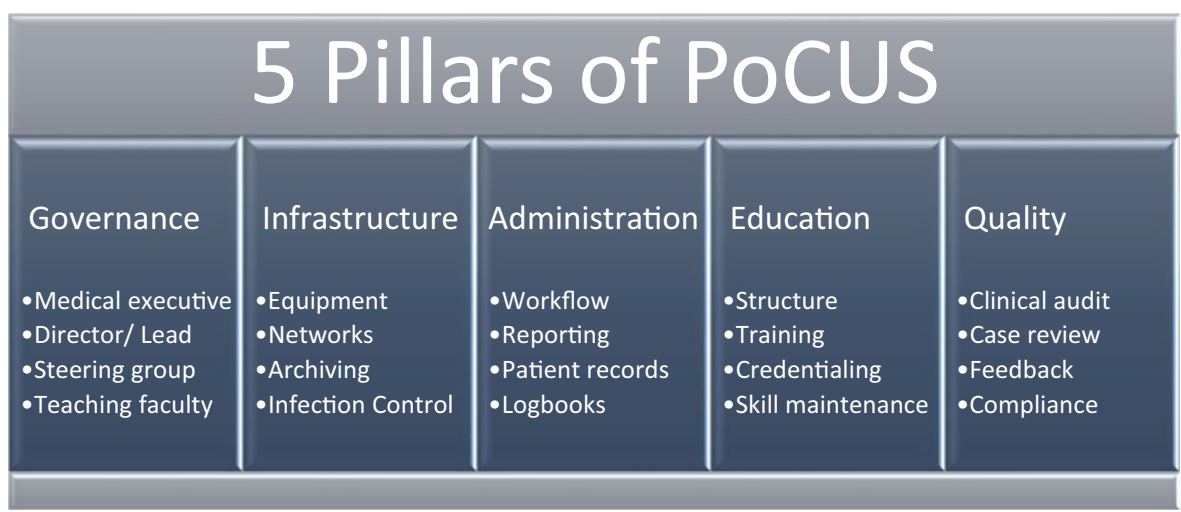

Figure 1: Five Pillars of Point-of-Care Ultrasound.

design, ergonomics, screen size, infection control, battery life, Wi-Fi/network connectivity, the ability to work in harsh environments and budget constraints. Governmental regulations relating to diagnostic imaging equipment standards will also need to be considered. ${ }^{10}$

As part of the purchase of equipment, negotiating servicing and warranty agreements are essential. We suggest a minimum 5 -year warranty be included in any purchase contract, including annual service maintenance and software upgrades. Infection control processes must be implemented to comply with Australasian Standards (e.g. AS/NZS 4187). These require highlevel disinfection of transducers for many examinations. ${ }^{11}$ Local procedures must incorporate transducer disinfection equipment, staff training, appropriate cleaning/storage zones and record keeping.

Hospital information technology is vital to the success of a PoCUS program. ${ }^{12}$ Digital Imaging and Communications in Medicine (DICOM) compatibility and Wi-Fi connectivity is the most ideal for image transfer to PACS. Local area network (LAN) image transfer via a data cable is recommended as a backup. It is important that the governance group develop institutional policy relating to approved PoCUS equipment, information technology, networks and image archiving. ${ }^{5,6}$ The ability to archive PoCUS studies according to local and international guidelines for patient information and privacy protection is paramount. ${ }^{12}$ Access to archived PoCUS images facilitates a complete patient imaging record for medicolegal and clinical quality assurance purposes. ${ }^{13}$ It should be noted that some handheld and smartphone ultrasound devices may be susceptible to issues with information security, image archiving and patient privacy. ${ }^{14} \mathrm{~A}$ challenge in this area is that infrastructure and PACS systems are expensive and can represent an element of infrastructure duplication within a hospital network. Cooperation between traditional ultrasound users (Radiology and Cardiology) giving PoCUS users access to store the images on existing PACS systems is one possible solution. ${ }^{8}$
Various other archival options are available, including web or cloud-based archiving, but patient privacy and security must be carefully considered.

\section{Pillar 3: Administration}

Administrative processes are necessary to support the other pillars of governance, infrastructure, education and quality. This may include policy, scan documentation, patient records, certification, clinician logbooks, education delivery and quality review. Some larger hospitals administrate websites with educational materials for clinicians. These tasks may be executed by various people such as the steering group, educators or other support staff.

At a minimum, physicians should have a mechanism to document scan findings and archive images for medicolegal purposes. Some alternatives exist for PoCUS reports including Electronic Medical Record (EMR) forms, handwritten patient progress notes and report screens completed on the ultrasound machine archived with scan images. There are also dedicated PoCUS IT systems that incorporate PACS, EMR and other program functions. ${ }^{15}$

\section{Pillar 4: Education}

Ultrasound is a complex motor skill, and PoCUS education involves ultrasound skill development, competency assessment, credentialing, ongoing education and skill maintenance. ${ }^{8,16}$ Our experience is that optimal training is provided in the context of robust program structure and stratification of skills into scan modules that enable skill progression and credentialing. A range of PoCUS competency standards has been defined by colleges and other medical bodies for various specialties.

PoCUS education and training may be achieved in many ways. The ideal educational leadership model is supported with qualified personnel with PoCUS experience. Protected teaching time, appropriate resourcing and administrative support will ensure the consistency of outcomes. Physicians in this role would ideally hold more than basic PoCUS credentials, such as 
fellowship or graduate diploma. Sonographers can also provide valuable leadership and education in PoCUS. ${ }^{8}$ In our experience, it is invaluable for departments utilising PoCUS to develop good relationships with Radiology and Cardiology, enabling review of difficult cases and ongoing learning. In settings where less experienced staff are in leadership roles, strong investment in self-education, upskilling and networking is required. In cases where there is a no local expertise or dedicated time, PoCUS education may be outsourced (e.g. external private ultrasound courses or privately contracted sonographers providing in-house training). In these situations, the close direction of a governance group is required to ensure proper clinical integration of PoCUS education and that the other pillars are adequately addressed.

\section{Pillar 5: Quality}

Quality audit processes should be integrated into the PoCUS program and are closely tied to the pillars of governance and education. Regular clinical auditing and clinician feedback are vital to education and quality. Clinical audit and case review are also an essential function of program governance. An auditing system is recommended, to enable consistent feedback to physicians by those reviewing scans. A scoring or traffic light system is a simple method that can provide quality feedback to clinicians via logbooks. ${ }^{8,13}$ Skill maintenance is another aspect of quality. It should be noted there is presently limited evidence, and no international consensus, about the number of scans required for attaining competency or the number of scans per annum required to maintain skills. Current guidelines of relevant medical bodies should be followed for PoCUS educational standards and competency assessment. ${ }^{17}$

\section{Conclusion}

The pillars of governance, infrastructure, administration, education and quality are vital to patient safety in hospital-based PoCUS training and credentialing programs. It is time to establish these pillars to ensure the best practice in PoCUS use.

\section{Disclosure statement}

AMW Casual tutor for Queensland University of Technology and Zedu Ultrasound Training Solutions.

\section{Authors declaration}

All contributing authors declare they have contributed equally to the development of this manuscript and agree with the content.

\section{References}

1 Kendall J, Hoffenberg S, Smith R. History of emergency and critical care ultrasound: the evolution of a new imaging paradigm. Crit Care Med 2007;35(5):126-30.
2 Moore CL, Copel JA. Point-of-care ultrasonography. $N$ Engl J Med 2011; 364: 749-57.

3 Stawicki SP, Bahner DP. Modern sonology and the bedside practitioner: evolution of ultrasound from curious novelty to essential clinical tool. Eur J Trauma Emerg Surg 2015; 41: 457-60.

4 Narula J, Chandrashekhar Y, Braunwald E. Time to add a fifth pillar to bedside physical examination: inspection, palpation, percussion, auscultation, and insonation. JAMA Cardiol 2018; 3: 346-50.

5 ACEP Policy Statement. Ultrasound guidelines: emergency, pointof-care and clinical ultrasound guidelines in medicine. Ann Emerg Med 2017; 69: e27-54.

6 Strony R, Marin J, Bailitz J, Dean A, Blaivas M, Tayal V, et al. Systemwide clinical ultrasound program development: an expert consensus model. WestJEM 2018; 19(4): 649-53.

7 Moore C. Safety Considerations in Building a Point-of-Care Ultrasound Program. Perspectives on Safety 2018. Available from: https://psnet.ahrq.gov/perspectives/perspective/251/safety-considera tions-in-building-a-point-of-care-ultrasound-program?q=moore+pr ogram. Accessed 27-August-2018.

8 Cormack C, Coombs P, Guskich K, Blecher GE, Goldie N, Ptasznik R. A collaborative model for training and credentialing point of care ultrasound: six year experience and quality outcomes. JMIRO 2018; 62: 330-6.

9 Moore G. Cramming more components onto integrated circuits. Electronics 1965; 38(8): 114-7.

10 Department of Health (2018). Equipment, Medicare and Diagnostic Imaging. Available from: http://www.health.gov.au/internet/ma in/publishing.nsf/Content/capsensdi. Accessed 30 October 2018.

11 Basseal JM, Westerway SC, Juraja M, van de Mortel T, McAuley TE, Rippey J, et al. Guidelines for reprocessing ultrasound transducers. Australas J Ultrasound Med 2017; 20: 30-40.

12 Au A, Adhikari S, Slovis B, Sachs PB, Lewiss RE. Hospital information technology is critical to the success of a point-of-care ultrasound program. Am J Emerg Med 2018; pii: S0735-6757(18) 30642-9. https://doi.org/10.1016/j.ajem.2018.08.007. [Epub ahead of print].

13 Byrne M, Geria R, Kummer T, Leech S, Lewiss R, Noble V, et al. Emergency ultrasound: workflow white paper. ACEP. Available from: https://www.acep.org/how-we-serve/sections/emergencyultrasound/running-a-program/\#sm.000922c01166fdmb10zlqzauh6 wkh. Accessed 29 August 18.

14 Physicians ACoE. Appropriate Use Criteria for Handheld/Pocket Ultrasound Devices; 2018. Available from: https://www.acep.org/ patient-care/policy-statements/appropriate-use-criteria-for-handhe ldpocket-ultrasound-devices/\#sm.000flimal141ef0lv342elif212ec

15 Lewiss R, Cook J, Sauler A, Avitabile N, Kaban NL, Rabrich J, et al. A workflow taskforce affects emergency physician compliance for point-of-care ultrasound documentation and billing. Crit Ultrasound J 2016; 8: 5.

16 Nicholls D, Sweet L, Hyett J. Psychomotor skills in medical ultrasound imaging. An analysis of the core skill set. J Ultrasound Med 2014;33:1349-52.

17 ASUM Position Statement. Minimum education and training requirements for ultrasound practitioners. AJUM 2017; 20(3): $132-5$. 